\title{
Temporal expectation weights visual signals over auditory signals
}

\author{
Melisa Menceloglu ${ }^{1}$ (D) $\cdot$ Marcia Grabowecky ${ }^{1,2} \cdot$ Satoru Suzuki $^{1,2}$
}

Published online: 28 June 2016

(C) Psychonomic Society, Inc. 2016

\begin{abstract}
Temporal expectation is a process by which people use temporally structured sensory information to explicitly or implicitly predict the onset and/or the duration of future events. Because timing plays a critical role in crossmodal interactions, we investigated how temporal expectation influenced auditory-visual interaction, using an auditory-visual crossmodal congruity effect as a measure of crossmodal interaction. For auditory identification, an incongruent visual stimulus produced stronger interference when the crossmodal stimulus was presented with an expected rather than an unexpected timing. In contrast, for visual identification, an incongruent auditory stimulus produced weaker interference when the crossmodal stimulus was presented with an expected rather than an unexpected timing. The fact that temporal expectation made visual distractors more potent and visual targets less susceptible to auditory interference suggests that temporal expectation increases the perceptual weight of visual signals.
\end{abstract}

Keywords Temporal expectation · Auditory · Visual · Congruity effect

Electronic supplementary material The online version of this article (doi:10.3758/s13423-016-1109-3) contains supplementary material, which is available to authorized users.

Melisa Menceloglu

mencel@u.northwestern.edu

1 Department of Psychology, Northwestern University, 2029 Sheridan Road, Evanston, IL 60208, USA

2 Interdepartmental Neuroscience Program, Northwestern University, Evanston, IL, USA
Temporal expectation is a process by which people use temporally structured sensory or motor information to predict the onset or duration of behaviorally relevant events. Temporal expectation is updated constantly and can influence perception, motivation, action, and learning (for reviews, see Coull \& Nobre, 2008; Nobre, Correa, \& Coull, 2007). The updating of temporal expectation can be either automatic (exogenous) or controlled (endogenous). In a typical experimental paradigm investigating exogenous temporal expectation, the temporal structures of events are manipulated without providing participants with explicit information regarding the temporal manipulations. For example, in a foreperiod paradigm (Niemi \& Näätänen, 1981), the duration of the interstimulus interval (ISI) between the cue and the target is manipulated. When the ISI is of fixed duration, the target onset is completely predictable from the onset of the cue, resulting in strong temporal expectation. In contrast, when the ISI is randomly varied, the target onset is less predictable, resulting in weak temporal expectation. Studies using the foreperiod paradigm have revealed that responses to targets become faster as temporal expectation becomes stronger (Lawrence \& Klein, 2013; Thomaschke, Kiesel, \& Hoffmann, 2011). In a typical experimental paradigm investigating endogenous temporal expectation, such as the temporal-cueing paradigm (Coull \& Nobre, 1998), a cue conveys the likely onset time of a target event, similar to an endogenous spatial-cueing paradigm in which a cue conveys the likely spatial location of a target event (Posner, 1980). As in spatial-cueing studies, temporalcueing has demonstrated that the processing of stimuli that appear at a cued time is enhanced (relative to those appearing at a different time), as indicated by faster responses and increased neural activation in sensory and motor cortices (e.g., Correa, Lupiáñez, Milliken, \& 
Tudela, 2004; Correa, Lupiáñez, \& Tudela, 2006; Lange, Krämer, \& Röder, 2006).

Temporal expectation has been shown to influence the behavioral responses to stimuli presented through different sensory modalities (e.g., visual, auditory, and tactile; Nobre et al., 2007), but it is less clear whether the effects of temporal expectation are modality-specific or amodal. For example, if one expects a visual stimulus to occur at a certain time, would facilitation at the expected time be limited to the processing of visual stimuli, or would the processing of stimuli through other modalities also benefit from being presented at the time expected for the visual stimulus? Lange and Röder (2006) used a modified version of a temporal-cueing paradigm in which participants were instructed to expect a specific cueto-stimulus interval — short or long - and a specific modality - auditory or tactile - to be presented more frequently in each block of trials. Responses were speeded and early auditory and somatosensory components of scalp-recorded eventrelated potentials were enhanced on trials in which a stimulus was presented at the expected time, regardless of whether the stimulus was presented in the expected or the unexpected modality. This result suggests that temporal expectation facilitates perceptual processing independently of modality. However, using a similar experimental design with visual and tactile stimuli, Mühlberg, Oriolo, and Soto-Faraco (2014) showed that, whereas the processing of stimuli in the expected modality benefited from occurring at the expected time, as indicated by faster responses, the processing of stimuli in the unexpected modality did not. This result suggests that temporal expectation facilitates perceptual processing in a modality-specific manner. The findings of modality independence versus modality specificity may depend on the stimuli, methods, or other experimental details. For instance, the two studies discussed above used different modality pairings (auditory-tactile vs. visual-tactile) and different pairs of temporal expectation intervals (600 or 1,200 ms vs. 1,000 or $2,500 \mathrm{~ms}$ ). Because sensory experience is nearly always multisensory, instead of focusing on whether temporal expectation is modality-specific using unimodal stimuli, we might gain significant insights into the mechanisms of temporal expectation and their roles in multisensory processing by investigating the effects of temporal expectation on crossmodal interactions using multimodal stimuli.

In the present study, we investigated the effects of temporal expectation on auditory-visual interactions. The strength of a crossmodal influence can be measured as the magnitude of a relevant crossmodal congruity effect (e.g., Orr \& Weissman, 2009; Weissman, Warner, \& Woldorff, 2004). The rationale is that when participants are instructed to respond to a target stimulus presented in a specific modality (e.g., the visual modality) while either a congruent or an incongruent stimulus is simultaneously presented by means of another modality (e.g., the auditory modality), the difference in performance between the congruent and incongruent conditions would indicate the degree of obligatory influence from the ignored modality. For example, if signals from the to-be-ignored modality were completely filtered out, performance would be identical in the congruent and incongruent conditions. The more the signals from the to-be-ignored modality influenced the responses to the signals from the attended modality, the worse performance would be in the incongruent relative to the congruent condition. Thus, a crossmodal congruity effect, defined as the incongruent-trial response time (RT) minus the congruent-trial RT in a speeded-response task, can provide a measure of how strongly the stimuli from the to-be-ignored modality obligatorily influence responses to the stimuli from the attended modality.

We used spoken and written letters as our multisensory stimuli because they are behaviorally relevant and have been shown to crossmodally interact on the basis of their overlearned auditory-visual correspondence (e.g., Larsen, McIlhagga, Baert, \& Bundesen, 2003; for a review, see Spence, 2011). For example, Larsen and colleagues (2003) simultaneously presented congruent or incongruent spoken and written letters. Participants had to report the written letter, the spoken letter, or both, depending on the condition. Regardless of the condition, performance was superior on the congruent relative to the incongruent trials. Using a similar behavioral paradigm and recording magnetoencephalography (MEG), Raij, Uutela, and Hari (2000) identified multisensory cortical networks that may mediate the integration of letterrelated information from the auditory and visual modalities. We thus expected that the processing of spoken and written letters would strongly interact, to generate robust auditoryvisual congruity effects. We therefore investigated how temporal expectation influenced this auditory-visual interaction by examining how expectation modulated the strength of the crossmodal congruity effect between spoken and written letters.

In our study, participants received a modality cue indicating whether a target letter would be presented visually or auditorily. The task was to identify the letter in the cued modality as rapidly and as accurately as possible. Each target was simultaneously accompanied by either a congruent or an incongruent letter presented via the other modality. Temporal expectation was block-wise manipulated by varying the relative probability of a bimodal stimulus presented at either a short or a long interval after the modality cue (without informing participants of the manipulation). In the shortinterval-dominant block of trials, each bimodal stimulus was frequently presented following a short interval, and infrequently presented following a long interval after the modality cue. In the long-interval-dominant block of trials, each bimodal stimulus was frequently presented following the long interval and infrequently presented following the short interval after the modality cue. The short-interval trials presented 
the critical condition, because the crossmodal stimuli appeared with an expected timing in the short-intervaldominant block - the expected-timing condition-and appeared with an unexpected timing in the long-intervaldominant block - the unexpected-timing condition. Note that long-interval trials were uninformative, because the longinterval stimulus was predictable even in the short-intervaldominant block; that is, if the stimulus did not appear after the short interval, it necessarily had to appear after the long interval. Consequently, participants could reorient temporal expectation upon not receiving the expected short-interval stimulus (Correa et al., 2004; Coull \& Nobre, 1998).

We expected to find a main effect of temporal expectation, in that RTs would be faster in the expectedtiming than in the unexpected-timing condition. We also expected to find a main effect of crossmodal congruity, in that RTs would be faster on the auditory-visual congruent than on the incongruent trials (e.g., Weissman et al., 2004). The critical question was how temporal expectation would modulate the crossmodal congruity effect. There were four possible outcomes, given that the crossmodal congruity effect was measured in two directions, auditory-to-visual (RTs to visual targets influenced by concurrent auditory stimuli) and visual-toauditory (RTs to auditory targets influenced by concurrent visual stimuli). First, both the auditory-to-visual and visual-to-auditory congruity effects might be increased in the expected-timing relative to the unexpected-timing condition. Such a bidirectional increase in the congruity effect would suggest that temporal expectation enhances crossmodal interactions, in the sense that it becomes more difficult to selectively attend to signals from one modality at the time of temporal expectation. Second, both the auditory-to-visual and visual-to-auditory congruity effects might be reduced in the expected-timing relative to the unexpected-timing condition. Such a bidirectional reduction in the congruity effect would suggest that temporal expectation facilitates focusing on the attended modality by reducing any influences from ignored modalities. Third, the auditory-to-visual congruity effect might be increased, but the visual-to-auditory congruity effect reduced, in the expected-timing relative to the unexpected-timing condition. Such a pattern of results would suggest that temporal expectation increases the perceptual weighting of auditory signals, allowing auditory processing to more strongly influence and be less strongly influenced by visual signals. The fact that the auditory modality typically dominates over the visual modality in temporal processing (e.g., Chen \& Yeh, 2009; Lukas, Philipp, \& Koch, 2014; Ortega, GuzmanMartinez, Grabowecky, \& Suzuki, 2014) might favor this outcome. Fourth, the auditory-to-visual congruity effect might be reduced, but the visual-to-auditory congruity effect increased, in the expected-timing relative to the unexpected-timing condition. Such a pattern of results would suggest that temporal expectation increases the perceptual weighting of visual signals, allowing visual processing to more strongly influence and be less strongly influenced by auditory signals.

\section{Method}

\section{Participants}

Thirty-one Northwestern University undergraduate students participated in the experiment. All had normal or correctedto-normal vision and normal hearing. Participants gave informed consent and were treated according to the guidelines of the Institutional Review Board at Northwestern University. Participants received partial course credit for their participation, which lasted approximately $1 \mathrm{~h}$. The data from two participants were excluded from the analyses; one participant failed to use the correct response keys, and the other did not pay attention to the task. The final sample included 29 participants ( 17 female, 12 male) between the ages of 18 and 21 years $(M=19, S D=0.98)$. The majority of them were right-handed (26 right-handed) and native English speakers (20 native English speakers).

\section{Stimuli and procedure}

The experiment was run on a 13-in., 2.3-GHz MacBook Pro and coded in MATLAB (Version R2013b) using the Psychophysics Toolbox extensions (Version 3.10.11; Brainard, 1997; Kleiner, Brainard, \& Pelli, 2007; Pelli, 1997). All visual stimuli were black $\left(1.2 \mathrm{~cd} / \mathrm{m}^{2}\right)$ and were presented at the center of the display monitor on a gray background $\left(73 \mathrm{~cd} / \mathrm{m}^{2}\right)$.

Each trial started with the presentation of an auditory or a visual cue, indicating the relevant target modality for the trial. The visual cue consisted of a row of three asterisks $\left(2^{\circ} \times 0.6^{\circ}\right.$ of visual angle) surrounded by a black rectangular frame $\left(19^{\circ} \times 14^{\circ}\right.$ of visual angle). The frame was present throughout the experiment, except during the intertrial interval (ITI). The auditory cue consisted of a 400-ms pure tone $(4410 \mathrm{~Hz})$ presented together with the rectangular frame. Either cue was followed by the presentation of a fixation $\operatorname{dot}\left(0.35^{\circ}\right.$ of visual angle) that lasted either $400 \mathrm{~ms}$ (short interval) or $1,200 \mathrm{~ms}$ (long interval). In the shortinterval-dominant block, the short interval was used $80 \%$ of the time, and the long interval was used $20 \%$ of the time. In the long-interval-dominant block, the probabilities were reversed. Following the short or long fixation interval, a bimodal target-distractor pair was presented for $350 \mathrm{~ms}$. 
Each target-distractor pair consisted of two letters- "B" and $\mathrm{B}$, "D" and $\mathrm{D}$, "B" and $\mathrm{D}$, or " $\mathrm{D}$ " and $\mathrm{B}$ - one presented auditorily and the other presented visually $\left(0.65^{\circ} \times\right.$ $0.83^{\circ}$ of visual angle). The letters (font size 48 ) and asterisks (font size 60) used for the visual stimuli were in Calibri font. All auditory stimuli were binaurally presented over headphones (60 dB SPLA). The auditory stimulus consisted of a female American-English speaker pronouncing the letter B or D. On congruent trials, the bimodal pair included the same letters (e.g., a visual B presented with a spoken /bee/); on incongruent trials, the bimodal pair included the opposite letters (e.g., a visual B presented with a spoken /dee/). The target-distractor pair was followed by another fixation display indicating the 1,650-ms response window. The next trial began after a 1,500-ms ITI from the end of the response window (Fig. 1).

Participants were seated approximately $65 \mathrm{~cm}$ away from the screen. They responded as to whether the letter of the cued modality was B or D by using the index and middle fingers of their dominant hand. The " $\mathrm{B}$ " response was mapped onto the "G" key, and the " $\mathrm{D}$ " response was mapped onto the adjacent " $H$ " key. These response keys were marked " $B$ " and " $D$," respectively. Participants rested their fingers on the response keys and were asked to maintain central fixation throughout the experiment and to respond as quickly and accurately as possible.

The experiment consisted of two blocks - a shortinterval-dominant block and a long-interval-dominant block-with a total of 560 trials (280 trials per block); 14 participants completed the short-interval-dominant block first, and 15 completed the long-interval-dominant block first. There were 35 trials for each of the eight trial types, consisting of the eight possible combinations of temporal expectation (expected [80\%] or unexpected [20\%]), target modality (auditory [50\%] or visual [50\%]), and crossmodal congruity (congruent [50\%] or incongruent [50\%]). Within a block, trials were presented in a random order. Short breaks were given between blocks and halfway through each block. A practice session was given at the beginning of the experiment, consisting of two blocks (given in the order in which they would be given during the experimental session) with a total of 32 trials. Responses were not recorded during the practice session.

\section{Data acquisition and analysis}

RT and accuracy data were recorded for each trial. Only the correct-response data were included in the RT analysis. The critical data came from the short-interval trials, in which the target was presented with the expected timing in the shortinterval-dominant block and with the unexpected timing in the long-interval-dominant block. An analysis of the data from the long-interval trials is presented in the Supplemental Material.

A three-way repeated measures analysis of variance (ANOVA) was performed, with RTs and proportions correct as the dependent variables, and Temporal Expectation (expected vs. unexpected), Target Modality (auditory vs. visual),

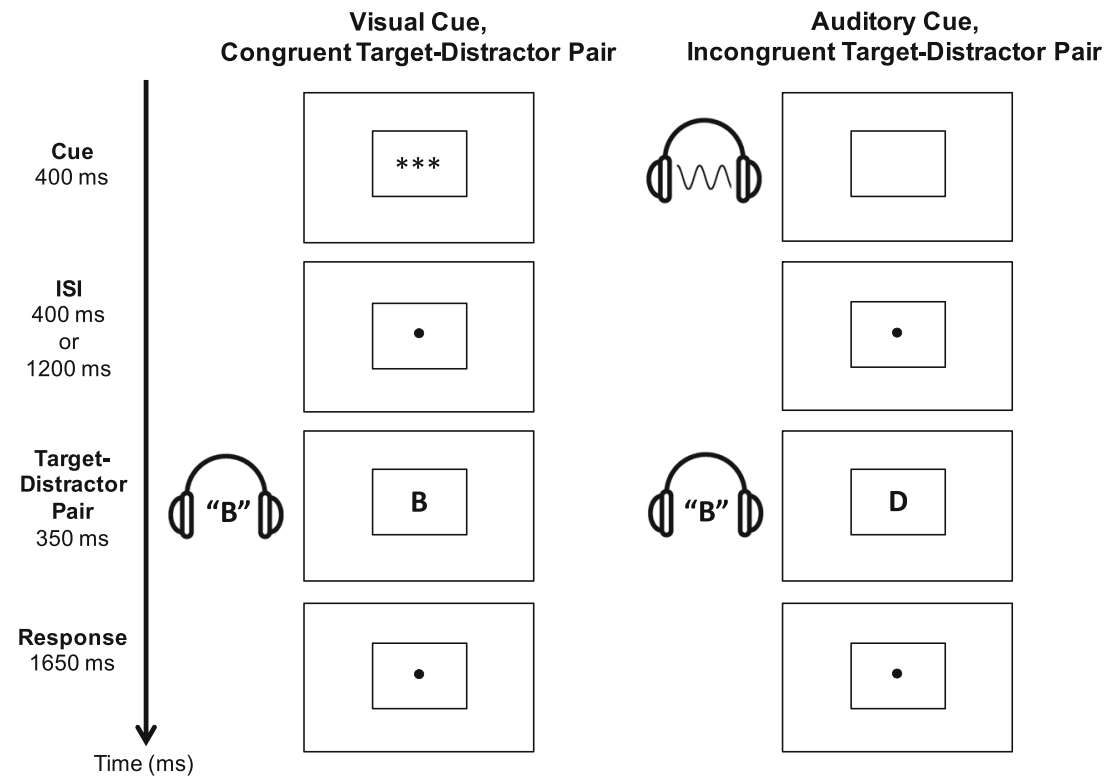

Fig. 1 Experimental task: A trial with a visual cue and a congruent target-distractor pair is shown on the left; a trial with an auditory cue and an incongruent target-distractor pair is shown on the right. The correct response would be "B" for both examples 
and Crossmodal Congruity (congruent vs. incongruent) as the within-subject factors.

\section{Results}

The overall response accuracy was high $(M=.942, S E=$ $.011)$. The only significant effect on accuracy was the main effect of crossmodal congruity; participants were more accurate on the congruent trials $(M=0.984 ; S E=0.006)$ than on the incongruent trials $(M=.900, S E=.016), F(1,28)=$ $46.1, p<.001, \eta_{\mathrm{p}}{ }^{2}=.62$. This result suggested no speedaccuracy trade-off, since the RTs were also shorter on congruent trials (see below).

RT analyses revealed a significant main effect of temporal expectation; participants responded faster on the expectedtiming trials $(M=883, S E=32)$ than on the unexpectedtiming trials $(M=946, S E=29), F(1,28)=10.32, p=.003$, $\eta_{\mathrm{p}}{ }^{2}=.27$ (Fig. 2, lower panel, where the darker bars are generally shorter than the lighter bars). This confirmed the effectiveness of our manipulation of temporal expectation. We also observed a significant main effect of crossmodal congruity; participants responded faster on the congruent trials $(M=846, S E=26)$ than on the incongruent trials $(M=$ 983, $S E=32), F(1,28)=106.08, p<.001, \eta_{\mathrm{p}}{ }^{2}=.79$ (Fig. 2, lower panel, where the left bar is shorter than the right bar for each pair). This confirmed that our crossmodal congruity manipulation was also effective. A significant main effect of target modality was also apparent; participants responded faster on the visual-target trials $(M=866, S E=31)$ than on the auditory-target trials $(M=964, S E=28), F(1,28)=55, p<$ $.001, \eta_{\mathrm{p}}{ }^{2}=.66$ (see Fig. 2, lower panel, where the bars are generally shorter in the right than in the left panel). None of the two-way interactions were significant (all $p s>.3$ ).

The crucial three-way interaction between temporal expectation, crossmodal congruity, and target modality was significant, $F(1,28)=19.72, p<.001, \eta_{\mathrm{p}}{ }^{2}=.41$. This interaction elucidates the four hypothetical outcomes discussed in the introduction: How do the crossmodal influences in the visual-to-auditory and auditory-to-visual directions, as

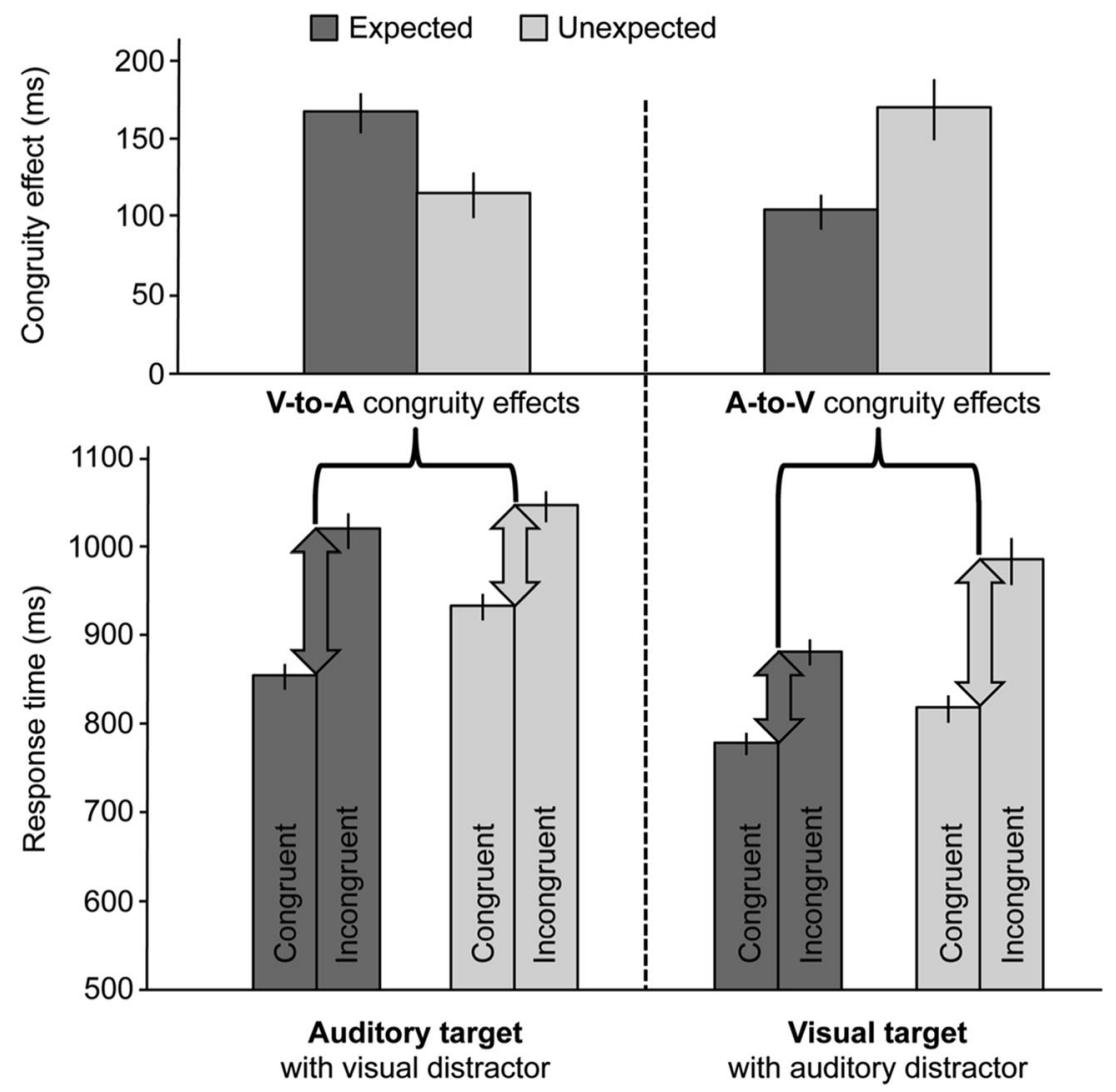

Fig. 2 In the lower panels, mean response times are shown for identifying auditory (left panel) or visual (right panel) targets paired with congruent or incongruent crossmodal distractors, presented with the expected (darker bars) or the unexpected (lighter bars) timing. The arrows in the left panel indicate the visual-to-auditory congruity effects for the auditory targets, whereas the arrows in the right panel indicate the auditory-to-visual congruity effects for the visual targets. These

crossmodal congruity effects are plotted separately in the upper panels. Note that temporal expectation increases the visual-to-auditory congruity effect (left panel) but reduces the auditory-to-visual congruity effect (right panel). Error bars represent \pm 1 standard error of the mean, adjusted for within-participants comparisons. Data from the short-interval trials are shown (see the main text). Data from the long-interval trials are presented in Fig. S1 in the supplementary material 
measured by the crossmodal congruity effects, depend on temporal expectation? The congruity effects (incongruent RT minus congruent RT) are illustrated with arrows in the lower panel of Fig. 2, and are separately plotted in the upper panel of that figure. It is clear from Fig. 2 that temporal expectation increased the visual-to-auditory RT congruity effect (upper left panel), whereas it reduced the auditory-to-visual RT congruity effect (upper right panel). Note that this two-way interaction is statistically equivalent to the aforementioned significant threeway interaction. Follow-up pairwise comparisons confirmed that the visual-to-auditory RT congruity effect was significantly larger on the expected-timing trials than on the unexpectedtiming trials, $t(28)=3.45, p=.002, d=0.64$, whereas the auditory-to-visual RT congruity effect was significantly smaller on the expected-timing trials than on the unexpected-timing trials, $t(28)=2.80, p=.009, d=0.52$. This pattern of result is consistent with the idea that temporal expectation increases the perceptual weighting of visual signals (relative to auditory signals), increasing the influences of irrelevant visual signals on auditory perception and decreasing the influences of irrelevant auditory signals on visual perception.

\section{Discussion}

Prior research has demonstrated that both perception through different sensory modalities and behavioral response are facilitated when an event occurs at an expected time (see the introduction). Prior research has also shown that the effect of temporal expectation can be either modality-specific (facilitation at the expected time only for a behavioral target in the expected modality) or modality-independent (facilitation at the expected time for a behavioral target in either an expected or an unexpected modality), depending on the pairing of sensory modalities and other stimulus factors (e.g., Lange \& Röder, 2006; Mühlberg et al., 2014). However, no prior study has investigated how temporal expectation might influence crossmodal interactions using multimodal stimuli, despite the fact that crossmodal integration crucially depends on timing (e.g., Holmes \& Spence, 2005).

We determined how temporal expectation modulated the influences from the ignored modality (visual or auditory) when people attended to a specific modality (auditory or visual). As was discussed in the introduction, we measured crossmodal influences as crossmodal congruity effects and considered four distinct possibilities. Specifically, if temporal expectation generally enhances multimodal influences, both auditory-to-visual and visual-to-auditory congruity effects should increase for stimuli at the expected time. If temporal expectation facilitates attention to a specific modality, both auditory-to-visual and visual-to-auditory congruity effects should then decrease for stimuli at the expected time. If temporal expectation increases the weighting of auditory signals, auditory-to-visual congruity effects should increase, but visual-to-auditory congruity effects should decrease, for stimuli at the expected time. Finally, if temporal expectation increases the weighting of visual signals, auditory-to-visual congruity effects should decrease, but visual-to-auditory congruity effects should increase, for stimuli at the expected time. Our results clearly support the final possibility (Fig. 2, upper panel).

An emphasis on visual processing at the time of temporal expectation may be consistent with behavioral demands. Although both auditory (e.g., rhythm) and visual (e.g., motion) signals provide useful information for setting up a temporal expectation for an upcoming event that may require timely action, executing the action at the expected moment may rely on visual information. For example, although the periodic sound and motion of a bouncing ball may both inform as to the time at which the ball will be within reach, visual information is crucial for catching the ball at the expected moment. The present results encourage further investigation of the mechanisms through which temporal processing modulates the weighting of sensory modalities, as well as the functional significance of this modulation.

Author note This study was supported by a National Institutes of Health grant (R01 EY021184).

\section{References}

Brainard, D. H. (1997). The psychophysics toolbox. Spatial Vision, 10, 433-436. doi:10.1163/156856897X00357

Chen, K., \& Yeh, S. (2009). Asymmetric cross-modal effects in time perception. Acta Psychologica, 130, 225-234. doi:10.1016/j. actpsy.2008.12.008

Correa, A., Lupiáñez, J., Milliken, B., \& Tudela, P. (2004). Endogenous temporal orienting of attention in detection and discrimination tasks. Perception \& Psychophysics, 66, 264-278. doi:10.3758 /BF03194878

Correa, A., Lupiáñez, J., \& Tudela, P. (2006). The attentional mechanism of temporal orienting: Determinants and attributes. Experimental Brain Research, 169, 58-68. doi:10.1007/s00221-005-0131-x

Coull, J. T., \& Nobre, A. C. (1998). Where and when to pay attention: The neural systems for directing attention to spatial locations and to time intervals as revealed by both PET and fMRI. Journal of Neuroscience, 18, 7426-7435.

Coull, J. T., \& Nobre, A. C. (2008). Dissociating explicit timing from temporal expectation with fMRI. Current Opinion in Neurobiology, 18, 137-144.

Holmes, N. P., \& Spence, C. (2005). Multisensory integration: Space, time and superadditivity. Current Biology, 15, R762-R764. doi:10.1016/j.cub.2005.08.058

Kleiner, M., Brainard, D., \& Pelli, D. (2007). What's new in Psychtoolbox-3? Perception, 36(ECVP Abstract Suppl.), 14.

Lange, K., Krämer, U. R., \& Röder, B. (2006). Attending points in time and space. Experimental Brain Research, 173, 130-140. doi:10.1007/s00221-006-0372-3

Lange, K., \& Röder, B. (2006). Orienting attention to points in time improves stimulus processing both within and across modalities. 
Journal of Cognitive Neuroscience, 18, 715-729. doi:10.1162 jocn.2006.18.5.715

Larsen, A., McIlhagga, W., Baert, J., \& Bundesen, C. (2003). Seeing or hearing? Perceptual independence, modality confusions, and crossmodal congruity effects with focused and divided attention. Perception \& Psychophysics, 65, 568-574.

Lawrence, M. A., \& Klein, R. M. (2013). Isolating exogenous and endogenous modes of temporal attention. Journal of Experimental Psychology: General, 142, 560-572. doi:10.1037/a0029023

Lukas, S., Philipp, A. M., \& Koch, I. (2014). Crossmodal attention switching: Auditory dominance in temporal discrimination tasks. Acta Psychologica, 153, 139-146.

Mühlberg, S., Oriolo, G., \& Soto-Faraco, S. (2014). Cross-modal decoupling in temporal attention. European Journal of Neuroscience, 39, 2089-2097.

Niemi, P., \& Näätänen, R. (1981). Foreperiod and simple reaction time. Psychological Bulletin, 89, 133-162. doi:10.1037/00332909.89.1.133

Nobre, A. C., Correa, A., \& Coull, J. T. (2007). The hazards of time. Current Opinion in Neurobiology, 17, 465-470.

Orr, J. M., \& Weissman, D. H. (2009). Anterior cingulate cortex makes 2 contributions to minimizing distraction. Cerebral Cortex, 19, 703 711. doi:10.1093/cercor/bhn119
Ortega, L., Guzman-Martinez, E., Grabowecky, M., \& Suzuki, S. (2014). Audition dominates vision in duration perception irrespective of salience, attention, and temporal discriminability. Attention, Perception, \& Psychophysics, 76, 1485-1502. doi:10.3758/s13414-014-0663-x

Pelli, D. G. (1997). The VideoToolbox software for visual psychophysics: Transforming numbers into movies. Spatial Vision, 10, 437-442. doi: $10.1163 / 156856897 \mathrm{X} 00366$

Posner, M. I. (1980). Orienting of attention. Quarterly Journal of Experimental Psychology, 32, 3-25. doi:10.1080 /00335558008248231

Raij, T., Uutela, K., \& Hari, R. (2000). Audiovisual integration of letters in the human brain. Neuron, 28, 617-625.

Spence, C. (2011). Crossmodal correspondences: A tutorial review. Attention, Perception, \& Psychophysics, 73, 971-995. doi:10.3758 /s13414-010-0073-7

Thomaschke, R., Kiesel, A., \& Hoffmann, J. (2011). Response specific temporal expectancy: Evidence from a variable foreperiod paradigm. Attention, Perception, \& Psychophysics, 73, 2309-2322. doi:10.3758/s13414-011-0179-6

Weissman, D. H., Warner, L. M., \& Woldorff, M. G. (2004). The neural mechanisms for minimizing cross-modal distraction. Journal of Neuroscience, 24, 10941-10949. 